\title{
Peer Specialists in Collaborative Care for Older Adults With Depression
}

\author{
Angela L. Rollins, Ph.D., Dana Frantz, M.S.W.
}

Community mental health has seen an explosion of peer specialists employed to support the recovery of persons with severe mental illnesses through such services as psychiatric illness management, medical self-management, and wellness programs. In 2008, we implemented a depression care management program for adults 65 or older in a primary care setting. The program resembled the Improving Mood-Promoting Access to Collaborative Treatment (IMPACT) program, including depression screening, brief problem-solving therapy, and consultation by a psychiatric prescriber in the event of depression nonresponse to therapy. However, the small primary care clinic where the program was embedded did not have a large enough population of older adults to support full IMPACT implementation, resulting in several modifications that may be relevant to other implementation efforts in small clinics. The program shifted to performing substantial screening and outreach to older adults in a variety of other settings, including small primary care practices that did not offer mental health care, senior service agencies, home health programs, and residential programs for older adults. Outreach therefore took on a substantial programmatic role, unlike the original IMPACT model. Peer specialists fulfilled this role in a cost-effective way, given that outreach is not billable to Medicare.

Peer specialists essentially opened the "front door" to the program for consumers. They provided formal and informal presentations on the signs of depression and the benefits of seeking treatment, conveying personal experience with depression and how the IMPACT program helped bring relief from symptoms and improve functioning. Prior to an initial appointment with other IMPACT staff members, virtually every consumer enrolled was screened formally for depression and engaged by a peer specialist. Peer specialists made home visits and phone calls to check in with consumers before or during initial engagement with the program to encourage them to keep appointments and discuss coping strategies, usually maintaining contact with ten to 20 consumers at any given time.

This is the author's manuscript of the article published in final edited form as:

Rollins, A. L., \& Frantz, D. (2015). Peer Specialists in Collaborative Care for Older Adults With Depression. Psychiatric Services, 66(9), 1000-1001. https://doi.org/10.1176/appi.ps.660904 
In some cases, the peer specialist provided transportation or other case management to make sure consumers were able to engage with the IMPACT program or other needed community services (such as housing support, meals, and medical care for chronic health conditions). Peer specialist assignments thus saved the more expensive therapist's time for services billable under Medicare. For one homebound elderly female consumer with no transportation or family nearby to get her to the clinic, the peer specialist was able to pick her up for appointments. The consumer was grateful not only for the transportation but also for the caring, personal experience of someone who was able to recover from depression, which gave her hope that depression was not an inevitable sign of aging. In another case, the peer was able to offer informal counseling and education to a husband who was having trouble understanding his wife’s behavior during a depressive episode.

The peer specialist also co-led depression relapse prevention groups with another provider for consumers who graduated from individual problem-solving treatment but still needed support to maintain their depression self-management skills.

The program employed two peer specialists and noted an obstacle that may be encountered by other programs seeking to use peers in this type of program. One peer specialist was an early graduate of the program and could easily connect with seniors who might have otherwise been skeptical of mental health services. The second peer specialist was already employed on an intensive case management team at the agency and readily disclosed his diagnosis of schizophrenia to consumers served by the intensive team. Within the IMPACT program, however, he became acutely aware of the stigma regarding a serious diagnosis like schizophrenia in the depressed older adult population and strategically avoided disclosing his particular diagnosis to the older adults. Instead, he described himself in vague terms as someone who had struggled with mental health "issues” and had a positive experience with recovery. This approach worked well but highlights the potential importance of matching peer specialist characteristics, such as age or diagnosis, to the client population being served.

Unfortunately, without the ability to bill for peer specialist services under Medicare, peer specialist time has since been decreased further to include services only for consumers with Medicaid, 
some of which include brief problem solving in the community for consumers who cannot easily make it to the clinic. This situation highlights the struggle to sustain innovative services for older adults under current Medicare reimbursement limitations. 